\title{
Grappes de cas asymptomatiques de COVID-19 en milieu familial au sein de la collectivité des Premières Nations dans le nord de la Saskatchewan, au Canada
}

\author{
Shree Lamichhane ${ }^{1}$, Sabyasachi Gupta ${ }^{1}$, Grace Akinjobi ${ }^{1}$, Nnamdi Ndubuka ${ }^{1,2 \star}$
}

Citation proposée : Lamichhane SR, Gupta S, Akinjobi G, Ndubuka N. Grappes de cas asymptomatiques de COVID-19 en milieu familial au sein de la collectivité des Premières Nations dans le nord de la Saskatchewan, au Canada. Relevé des maladies transmissibles au Canada, 2021;47(2):105-7.

https://doi.org/10.14745/ccdr.v47i02a01f

Mots-clés : COVID-19, asymptomatique, Première Nation, Canada, grappe de cas en milieu familial, transmission

\section{Introduction}

Un nouveau coronavirus (coronavirus du syndrome respiratoire aigu sévère 2 , SRAS-CoV-2), causant une grappe de cas d'infections respiratoires, est apparu pour la première fois à Wuhan, en Chine, en décembre 2019. L'éclosion s'est étendue rapidement dans le monde entier et, à partir du 7 décembre 2020, un total de 67440864 cas ont été confirmés dans 191 pays, entraînant 1541661 décès. De nombreux symptômes de la maladie à coronavirus de 2019 (COVID-19) ont été signalés. Les symptômes varient entre légers et graves, et peuvent apparaître 2 à 14 jours après l'exposition au virus. Récemment, on a observé que les cas asymptomatiques ou présymptomatiques constituent ce qui peut être une grande partie de toutes les infections par la COVID-19. Si ces cas ne peuvent être identifiés et isolés de manière appropriée afin de faciliter une intervention médicale, cela pourrait limiter l'efficacité des mesures de prévention de la transmission.

Nous signalons une grappe de cas de COVID-19 en milieu familial qui a commencé avec un cas paucisymptomatique et a conduit à deux cas asymptomatiques. Dans notre grappe de cas en milieu familial, cinq cas sur neuf (55\%) étaient présymptomatiques au moment du test de dépistage, tandis que deux cas (22\%) sont restés asymptomatiques tout au long de l'infection.

\section{Situation actuelle}

Depuis le début de la pandémie, la province de la Saskatchewan, au Canada, a signalé 11475 cas de COVID-19. Parmi ces cas, 910 provenaient des collectivités des Premières Nations de la Northern Inter-Tribal Health Authority (NITHA) dans le nord de la Saskatchewan (http://www.nitha.com/ - en anglais). Étant donné
Cette oeuvre est mise à la disposition selon les termes de la licence internationale Creative Commons Attribution 4.0

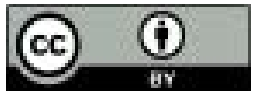

Affiliations

${ }^{1}$ Northern Inter-Tribal Health Authority, Prince Albert, SK

2 School of Public Health, University of Saskatchewan, Saskatoon, SK

\section{*Correspondance :}

nndubuka@nitha.com que les personnes asymptomatiques et présymptomatiques sont une source potentielle d'infection par la COVID-19 $(1,2)$, nous signalons une grappe de cas en milieu familial parmi les Premières Nations du nord de la Saskatchewan où l'infection a commencé avec un cas paucisymptomatique et a conduit à deux cas asymptomatiques. On reconnaît, de plus en plus, que les déterminants de la santé des Autochtones, comme le surpeuplement, la pauvreté, l'incidence de l'histoire des pensionnats indiens, les jeunes, la faiblesse de l'infrastructure de la santé publique, l'accès limité à des services de santé de qualité et l'augmentation du taux de comorbidité, peuvent aggraver les éclosions de maladies (3). Plus précisément, les logements surpeuplés peuvent entraîner une distanciation physique inefficace et des mesures inadéquates de lutte contre les infections avec une probabilité accrue de transmission de la COVID-19. II y a également un risque accru qu'il y ait une mauvaise santé mentale, des hospitalisations et de graves conséquences chez les personnes des Premières Nations immunodéficientes et atteintes d'une maladie chronique (4). Comme de nombreuses collectivités de Premières Nations sont maintenant touchées par les éclosions de COVID-19, le 
présent rapport fournit également les données nécessaires à l'élaboration et à l'application de stratégies de santé publique dans d'autres collectivités de Premières Nations.

Notre cas de référence (groupe d'âge de 20 à 29 ans) a contracté l'infection d'un contact étroit qui est retourné dans la collectivité d'une région à l'extérieur de la province où la transmission est élevée, et, ultérieurement, a développé un symptôme bénin (rhinite), qui a disparu en quelques jours. Deux jours plus tard, le cas de référence a assisté à un dîner familial où il semble qu'une nouvelle transmission s'est produite. Après avoir effectué la recherche des contacts, huit cas de plus ont été identifiés; trois du ménage du cas de référence et cinq d'un autre ménage à qui le cas de référence a rendu visite (figure 1). Le moment exact de l'exposition à la transmission n'a pas pu être déterminé parce que les personnes avec lesquelles le cas de référence était en contact vivaient dans des milieux surpeuplés et l'exposition était en cours. Aucune autre exposition possible n'a été identifiée qui pourrait être liée à cette infection par la COVID-19. Tous les patients positifs au COVID-19 et leurs contacts étroits ont été isolés conformément aux normes provinciales.

Le deuxième patient (groupe d'âge de 10 à 19 ans) et le troisième patient (groupe d'âge de 30 à 39 ans) du ménage 1 ont présenté des symptômes très légers (perte du goût et de l'odorat) pendant deux jours; cependant, le premier patient (groupe d'âge de 40 à 49 ans) n'a pas développé de symptômes. Du ménage 2, le sixième patient est tombé malade et a eu une pharyngite. Le cinquième patient (groupe d'âge de 30 à 39 ans, ayant un problème de santé chronique et préexistant) a signalé la perte de goût et de l'odorat, suivie de toux, d'essoufflement et de diarrhée. À mesure que l'état du patient s'aggravait, ce patient a été hospitalisé et a guéri dans deux semaines. Le septième patient (un nourrisson) est tombé malade et a développé de la fièvre et une toux; cependant, l'état du patient s'est amélioré sans intervention médicale. Le huitième patient (groupe d'âge de 20 à 29 ans), qui, au début, a obtenu un résultat négatif lors du dépistage de la COVID-19 basé sur la réaction en chaîne de la polymérase, a développé des symptômes (respiration sifflante et fièvre) dans les 12 jours suivant l'exposition et a reçu un résultat positif lors d'un nouveau test de dépistage de la COVID-19. Dans l'ensemble, trois patients du ménage 2 ont été déclarés asymptomatiques au moment du test de dépistage, dont un (le quatrième patient du groupe d'âge de 5 à 9 ans) n'a présenté aucun symptôme pendant la période d'isolement.

Dans notre grappe de cas en milieu familial, cinq cas sur neuf (55 \%) étaient présymptomatiques au moment du test de dépistage, tandis que deux cas (22 \%) n'ont développé aucun symptôme durant environ deux semaines de suivi. Notre cas de référence ne présentait que des symptômes légers et ne connaissait pas l'état accru des risques liés à la COVID-19, ce qui a ajouté de I'incertitude et a retardé la détection précoce et l'isolement. En dépit de ces préoccupations, six cas sur neuf n'ont présenté que des symptômes légers et ont guéri en recevant des soins médicaux minimaux, ce qui souligne la possibilité de confiner des cas de COVID-19 à l'extérieur de l'hôpital en fournissant une surveillance et des conseils appropriés. Étant donné que les collectivités rurales peuvent faire face à des défis différents liés à la COVID-19, selon l'endroit où elles se trouvent, chaque collectivité ainsi que les membres de la collectivité devraient évaluer leur sensibilité et leur vulnérabilité sociale uniques à la COVID-19 et y intervenir conformément aux mesures de santé publique. Les mesures pertinentes pour prévenir la transmission communautaire de la COVID-19 au sein de ces communautés vulnérables visent à éviter les déplacements non essentiels à l'extérieur de la collectivité et à limiter les interactions entre les personnes de différents ménages.

Figure 1 : L'historique de l'exposition de cas de référence et de ménage dans la grappe de cas en milieu familial

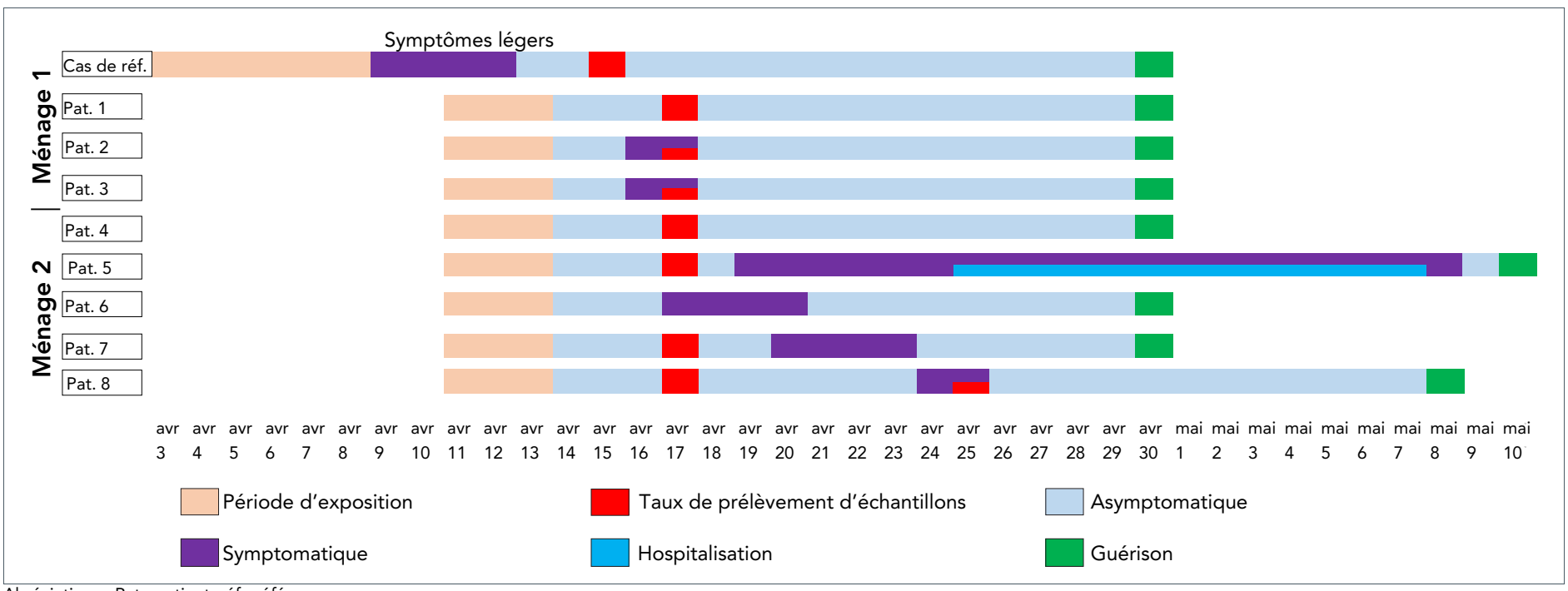

Abréviations : Pat., patient; réf., référence 


\section{Conclusion}

La détection précoce et l'isolement des patients symptomatiques de la COVID-19 qui font l'objet d'enquêtes de recherche de contacts efficaces constituent une stratégie importante de confinement de la maladie. Étant donné que la transmission asymptomatique et présymptomatique est biologiquement plausible $(1,2)$, une telle transmission pourrait limiter l'efficacité des mesures de contrôle $(2,5,6)$. Ce résumé des cas souligne l'importance de la détection précoce, de la recherche des contacts, du dépistage de tous les contacts proches, quels que soient les symptômes qu'ils présentent, et de l'isolement préventif de 14 jours des personnes provenant des zones à forte transmission qui retournent dans les collectivités, pour prévenir la propagation asymptomatique dans les collectivités éloignées. II souligne également le besoin d'un seuil minimal pour faire dépister les personnes présentant des symptômes très légers dans les 14 jours qui suivent leur retour des zones à forte transmission. La transmissibilité par des patients asymptomatiques ou présymptomatiques dans les cas de logements surpeuplés, comme ceux que l'on trouve souvent dans des collectivités éloignées, nordiques et autochtones, peut contribuer à des taux de transmission plus élevés.

\section{Déclaration des auteurs}

S. R. L. - Examen de la documentation, rédaction-ébauche S. G. - Analyse des données, rédaction-ébauche

G. A. - Rédaction-révision et édition

N. N. - Élaboration, conception, interprétation des données, examen critique

\section{Intérêts concurrents}

Aucun.

\section{Remerciements}

Les auteurs remercient la Northern Inter-Tribal Health Authority et les communautés partenaires pour leur travail acharné et leur contribution à cet article.

\section{Financement}

Aucun.

Le contenu de l'article et les points de vue qui y sont exprimés n'engagent que les auteurs et ne correspondent pas nécessairement à ceux du gouvernement du Canada.

\section{Références}

1. Rothe C, Schunk M, Sothmann P, Bretzel G, Froeschl G, Wallrauch C, Zimmer T, Thiel V, Janke C, Guggemos W, Seilmaier M, Drosten C, Vollmar P, Zwirglmaier K, Zange S, Wölfel R, Hoelscher M. Transmission of 2019-nCoV infection from an asymptomatic contact in Germany. N Engl J Med 2020 Mar;382(10):970-1. DOI PubMed

2. Zhang J, Tian S, Lou J, Chen Y. Familial cluster of COVID-19 infection from an asymptomatic. Crit Care 2020 Mar;24(1):119. DOI PubMed

3. Richardson KL, Driedger MS, Pizzi NJ, Wu J, Moghadas SM. Indigenous populations health protection: a Canadian perspective. BMC Public Health 2012 Dec;12(1):1098. DOI PubMed

4. Schiavo R, May Leung M, Brown M. Communicating risk and promoting disease mitigation measures in epidemics and emerging disease settings. Pathog Glob Health 2014 Mar;108(2):76-94. DOI PubMed

5. Wei WE, Li Z, Chiew CJ, Yong SE, Toh MP, Lee VJ. Presymptomatic Transmission of SARS-CoV-2-Singapore, January 23-March 16, 2020. MMWR Morb Mort Wkly Rep. 2020;69(14):411-5. https://www.cdc.gov/mmwr/volumes/69/ $\mathrm{wr} / \mathrm{mm} 6914 \mathrm{e} 1 . \mathrm{htm}$

6. Tian S, Hu N, Lou J, Chen K, Kang X, Xiang Z, Chen $\mathrm{H}$, Wang D, Liu N, Liu D, Chen G, Zhang Y, Li D, Li J, Lian $\mathrm{H}$, Niu S, Zhang L, Zhang J. Characteristics of COVID-19 infection in Beijing. J Infect. 2020;80(4):401-6. DOI PubMed 